\title{
QUADRATIC PERFORMANCE ANALYSIS OF SWITCHED AFFINE TIME-VARYING SYSTEMS
}

\author{
WENZHi LI ${ }^{a}$, CHI HUANG ${ }^{a, *}$, GUISHENG ZHAI ${ }^{b}$ \\ ${ }^{a}$ College of Mathematics \\ Taiyuan University of Technology, Taiyuan 030024, China \\ e-mail: huangchi@tyut.edu.cn \\ ${ }^{b}$ Department of Mathematical Sciences \\ Shibaura Institute of Technology, Saitama 337-8570, Japan
}

\begin{abstract}
We analyze quadratic performance for switched systems which are composed of a finite set of affine time-varying subsystems, where both subsystem matrices and affine vectors are switched, and no single subsystem has desired quadratic performance. The quadratic performance indexes we deal with include stability, tracking and $\mathcal{L}_{2}$ gain. We show that if a linear convex combination of subsystem matrices is uniformly Hurwitz and another convex combination of affine vectors is zero, then we can design a state-dependent switching law (state feedback) and an output-dependent switching law (output feedback) such that the entire switched affine system is quadratically stable at the origin. In the case where the convex combination of affine vectors is nonzero, we show that the tracking control problem can be posed and solved using a similar switching strategy. Finally, we consider the $\mathcal{L}_{2}$ gain analysis problem for the switched affine time-varying systems under state feedback.
\end{abstract}

Keywords: switched affine systems, time-varying systems, quadratic stabilization, tracking, $\mathcal{L}_{2}$ gain, switching law, differential LMIs, observers.

\section{Introduction}

1.1. Background and motivation. Switched systems are composed of a family of continuous-time or discrete-time subsystems and a switching law determining which subsystem should be activated at each time instant. Since many practical systems can be modeled as switched systems, there have been increasing interest and a large amount of results on stability analysis and control design of switched systems in the last two decades. For some progress and perspectives in the field of switched systems, see the survey papers by Branicky (1998), Liberzon and Morse (1999) or DeCarlo et al. (2000), the books by Liberzon (2003) or Sun and Ge (2005), and the references cited therein.

As also pointed out by Liberzon and Morse (1999), Liberzon (2003) or Feron (1996), there are three basic problems in the analysis and design of switched systems. The first one is to establish the conditions under which the switched system is stable under an arbitrary switching.

* Corresponding author
The second basic problem considers the case where both stable and unstable subsystems exist, and consists in identifying and designing switching strategies such that the system is stabilized. It is known that one of the main approaches is the (average) dwell time scheme together with piecewise Lyapunov functions (Allerhand and Shaked, 2011; Xiang and Xiao, 2014; Xiang, 2016). The third basic problem, which we consider in this paper, is to design a stabilizing switching law (strategy) for the case where each single subsystem is not stable. There are several approaches dealing with this issue, especially for switched linear systems. Pettersson and Lennartson (2002) used Lyapunov-like functions to partition the entire state space into different regions, and proposed LMI-based state-dependent switching laws, such that the switched system is stabilized. Wicks et al. (1998) and Feron (1996) showed that the existence of a stable convex combination of subsystem matrices implies the existence of a state-dependent switching rule that stabilizes the switched system along with a quadratic Lyapunov function that proves it. An extension to output-dependent 
switching for quadratic stabilizability was made with a robust detectability condition by Feron (1996). Zhai (2001) extended the results of Feron (1996) and Wicks et al. (1998) to the case of discrete-time switched linear systems, by describing the quadratic stabilizability condition as a nonnegative combination of subsystems' Lyapunov inequalities. Later, for switched linear systems with polytopic uncertainties (both continuous-time and discrete-time), quadratic stabilizability via state feedback was established by Zhai et al. (2003).

The results of Feron (1996) and Wicks et al. (1998) were further extended by Zhai (2012) to quadratic stabilizability analysis with $\mathcal{L}_{2}$ gain (in the name of $\mathcal{H}_{\infty}$ disturbance attenuation level) of switched linear systems, under the assumption that there is no single subsystem which is quadratically stable with $\mathcal{L}_{2}$ gain $\gamma$. In that context, assuming that there exists a convex combination of subsystems which is quadratically stable with $\mathcal{L}_{2}$ gain $\gamma$, the authors have designed a state-dependent switching law (state feedback) such that the entire switched system is quadratically stable with the same $\mathcal{L}_{2}$ gain. The switching law is established with a positive definite matrix, which guarantees quadratic stability with $\mathcal{L}_{2}$ gain $\gamma$ for the convex combination of subsystems. The authors also showed that when the number of subsystems is two, the existence of the convex combination of subsystems is not only sufficient but also necessary. The discussion was further broadened to the case of output-dependent switching law (output feedback) when the measurement output is available instead of the state.

Motivated by these works, this paper aims to extend the approach of convex combination of subsystems, proposed by Feron (1996), Wicks et al. (1998) and Zhai (2012), to quadratic performance analysis for switched affine time-varying systems. Different from switched linear time-invariant systems, we first assume that the subsystem matrices are time-varying, and furthermore a time-varying affine vector exists in the vector fields. To generalize the convex combination approach, we assume as before that the subsystems do not have common equilibrium states. Then, in addition to the difficulty of dealing with time-varying subsystems, the stabilization to a desired "equilibrium" state is difficult and even not easy to formulate. Needless to say, another performance analysis such as $\mathcal{L}_{2}$ gain analysis is more challenging.

For switched affine systems (SASs), there have been several good papers working on the analysis and design problems. Hetel and Fridman (2013) considered the stabilization problem for SASs with a sampled-data switching law. Deaecto and Santos (2015) as well as Deaecto (2016) dealt with state/output feedback switching function control design for continuous-time SAS, assuring global asymptotic stability of a desired equilibrium point and a guaranteed $H_{\infty}$ performance level. Trofino et al. (2009) and Scharlau et al. (2014) established a max-type composition switching rule such that the SASs converges to a desired "equilibrium" point globally asymptotically. Yoshimura et al. (2013) proposed an observer-based control design for SAS by using a switched quadratic Lyapunov function approach.

We have been working in this area, and proposed the idea of practical stability/stabilization together with several efficient switching laws (Xu and Zhai, 2005; Xu et al., 2008). Additionally, the idea of using stable convex combinations of subsystems has also been studied for a class of switched affine systems by Bolzern and Spinelli (2004). In this paper, we show that if a linear convex combination of subsystem matrices is uniformly Hurwitz and another convex combination of affine vectors is zero, then we can design a state-dependent switching law (state feedback) and an output-dependent switching law (output feedback) such that the entire switched affine system is quadratically stable at the origin. In the case where the convex combination of affine vectors is nonzero, we show that a class of tracking control problems can be well posed and solved using a similar switching strategy. Finally, we consider the $\mathcal{L}_{2}$ gain analysis problem for the switched affine time-varying systems under state feedback.

1.2. Notation and preliminaries. Throughout this paper, the superscript " $T$ " represents the transpose of a matrix, and the superscript " -1 " represents the inverse of a regular matrix. $W \succ 0(W \prec 0)$ means $W$ is symmetric and positive (negative) definite, and $W_{1} \succ W_{2}$ means $W_{1}-W_{2} \succ 0 . W \succeq 0(W \preceq 0)$ means $W$ is symmetric and nonnegative (non positive) definite, and $W_{1} \succeq W_{2}$ means $W_{1}-W_{2} \succeq 0$. A constant system matrix $A$ is Hurwitz if all its eigenvalues have negative real parts. For a time-varying vector $w(t)$, the notation $w(t) \in \mathcal{L}_{2}[0, \infty)$ means that it is square integrable in the sense of $\int_{0}^{\infty} w^{\top}(\tau) w(\tau) \mathrm{d} \tau<\infty$.

A time-varying symmetric matrix $W(t)$ is said to be uniformly positive definite if $W(t) \succ \alpha I$ holds for some positive scalar $\alpha$. Then, $W(t)$ is said to be uniformly negative definite if $-W(t)$ is uniformly positive definite. A time-varying system matrix $A(t)$ is uniformly Hurwitz if all real parts of its eigenvalues are less than a negative scalar.

Next, as a preliminary to later stability and $\mathcal{L}_{2}$ gain analysis, we state a well-known bounded real lemma (Skelton et al., 1998) for continuous-time linear time-varying (LTV) systems.

Lemma 1. Consider the linear time-varying (LTV) system

$$
\left\{\begin{array}{l}
\dot{x}(t)=A(t) x(t)+B(t) w(t), \\
z(t)=C(t) x(t)+D(t) w(t),
\end{array}\right.
$$

where $x(t), z(t), w(t)$ are the state, the controlled output and the disturbance input, respectively. 
1. When $w(t)=0(t \geq 0)$, the system (1) is uniformly asymptotically stable at the origin if and only if there exists a real matrix function $P(t)$ which is uniformly bounded and uniformly positive definite, such that

$$
\dot{P}(t)+A^{\top}(t) P(t)+P(t) A(t)
$$

is uniformly negative definite.

2. The system (1) is uniformly asymptotically stable at the origin and the $\mathcal{L}_{2}$ gain from $w$ to $z$ is less than a positive scalar $\gamma$ if and only if there exists a real matrix function $P(t)$ which is uniformly bounded and uniformly positive definite, such that

$$
\left[\begin{array}{rl}
\dot{P}(t)+A^{\top}(t) P(t) & +P(t) A(t)+C^{\top}(t) C(t) \\
B^{\top}(t) P(t) & +D^{\top}(t) C(t) \\
P(t) B(t)+C^{\top}(t) D(t) \\
-\gamma^{2} I+D^{\top}(t) D(t)
\end{array}\right]
$$

is uniformly negative definite.

\section{Problem formulation}

In this paper, we consider the switched system composed of a finite set of affine time-varying subsystems described by

$$
\left\{\begin{aligned}
\dot{x}(t) & =A_{\sigma_{1}(t)}(t) x(t)+b_{\sigma_{2}(t)}(t)+B(t) w(t), \\
z(t) & =C(t) x(t)+D(t) w(t), \\
y(t) & =E(t) x(t),
\end{aligned}\right.
$$

where $x(t) \in \mathbb{R}^{n}$ is the state ( $x_{0}$ is the initial state), $w(t) \in \mathbb{R}^{m}$ is the disturbance input taking values in $\mathcal{L}_{2}[0, \infty), z(t) \in \mathbb{R}^{p}$ is the controlled output, and $y(t) \in \mathbb{R}^{q}$ is the measurement output. Additionally, $\sigma_{1}(t): \mathbb{R} \rightarrow I_{\mathcal{N}_{1}}=\left\{1, \ldots, \mathcal{N}_{1}\right\}$ and $\sigma_{2}(t): \mathbb{R} \rightarrow I_{\mathcal{N}_{2}}=\left\{1, \ldots, \mathcal{N}_{2}\right\}$ are switching laws, which will be designed later. Thus, the matrix function $A_{\sigma_{1}(t)}(t)$ takes values in the finite set $\left\{A_{1}(t), \ldots, A_{\mathcal{N}_{1}}(t)\right\}$, and the vector function $b_{\sigma_{2}(t)}(t)$ takes values in the finite set $\left\{b_{1}(t), \ldots, b_{\mathcal{N}_{2}}(t)\right\}$. Here, $A_{i}(t), b_{j}(t), B(t), C(t), D(t), E(t)$ are time-varying matrices/vectors of appropriate dimensions, denoting the $(i, j)$-th subsystem $\mathcal{S}_{i j}$, and $\mathcal{N}_{1} \times \mathcal{N}_{2}$ is the number of subsystems.

Next, we give two definitions corresponding to the control problems we handle in this paper. The first is about quadratic stability/stabilization, and the second is about $\mathcal{L}_{2}$ gain analysis. In addition, without causing confusion, we use the term "state feedback" ("output feedback") to denote "state-dependent switching" ("output-dependent switching”) (Zhai et al., 2003; Zhai, 2012; Leth and Wisniewski, 2014).
Definition 1. The switched affine system (4) for which $w(t)=0$ is quadratically stabilizable via state feedback at the origin if there exist a positive definite function $V(x, t)=x^{\top} P(t) x$ with $P(t)$ being uniformly bounded and uniformly positive definite, a positive scalar $\epsilon$ and switching law $\left(\sigma_{1}(x, t), \sigma_{2}(x, t)\right)$ such that

$$
\dot{V}(x, t)<-\epsilon V(x, t)
$$

for all nonzero trajectories $x$ of the switched system (4). When the switching law depends on the measurement output $y$, i.e., taking the form of $\left(\sigma_{1}(y, t), \sigma_{2}(y, t)\right)$, if (5) holds, the switched affine system (4) is quadratically stabilizable via output feedback.

Definition 2. The switched affine system (4) is quadratically stabilizable with $\mathcal{L}_{2}$ gain $\gamma$ via state feedback at the origin if there exist a positive definite function $V(x, t)=x^{\top} P(t) x$ with $P(t)$ being uniformly bounded and uniformly positive definite, a positive scalar $\epsilon$ and switching law $\left(\sigma_{1}(x, t), \sigma_{2}(x, t)\right)$ such that

$$
\dot{V}(x, t)<-\epsilon V(x, t)-z^{\top} z+\gamma^{2} w^{\top} w
$$

for all $w(t) \in \mathcal{L}_{2}[0, \infty)$ and all nonzero trajectories $x$ of the switched system (4).

Remark 1. It is easy to see that the requirement (5) leads to exponential stability, which is an ideal property in practical systems. To verify the meaning of Definition 2, since (6) holds for any time instant with the same matrix function $P(t)$, we integrate it from 0 to arbitrary time instant $t$ to obtain

$$
\int_{0}^{t} z^{\top} z \mathrm{~d} \tau<V(x(0), 0)+\gamma^{2} \int_{0}^{t} w^{\top} w \mathrm{~d} \tau,
$$

which exactly implies the $\mathcal{L}_{2}$ gain $\gamma$.

If there is a common equilibrium state $x_{e}$ for all subsystems $\mathcal{S}_{i j}$, then by using a state transformation we obtain a switched linear system which has been studied extensively in the literature. Since any equilibrium state can be shifted to the origin, without losing generality, we consider stability of the switched affine system at the origin. Here we aim to use a combination of subsystems to propose a stabilizing switching law for the switched affine system. To make the switching problem non-trivial, throughout this paper we assume that there is no common equilibrium state for all subsystems $\mathcal{S}_{i j}\left(i=1, \ldots, \mathcal{N}_{1}\right.$; $\left.j=1, \ldots, \mathcal{N}_{2}\right)$. Moreover, we assume that when $w(t)=$ 0 , there is no single subsystem which is quadratically stable, and when $w(t) \neq 0$, there is no single subsystem which is quadratically stable with $\mathcal{L}_{2}$ gain $\gamma$. Otherwise, we can choose to activate certain subsystem for all time.

Compared with switched linear time-varying systems, the stabilization of switched affine time-varying 
systems is much more difficult due to the existence of affine vectors $b_{j}(t)\left(j=1, \ldots, \mathcal{N}_{2}\right)$, and even looks like unreasonable in some cases. However, in our previous work (Xu and Zhai, 2005; Xu et al., 2008), we have shown that even for switched integrator systems, where each subsystem does not have any equilibrium state, we can design a switching law such that the system is practically and asymptotically stable. This inspires us to consider more general switched affine systems, such as the ones in this paper, and to pursue various control performance criteria including tracking and the $\mathcal{L}_{2}$ gain property.

It is emphasized that the main feature of the system (4) different from the literature consists in introducing two switching laws, $\sigma_{1}(t)$ for the subsystem matrix $A_{i}(t)$ and $\sigma_{2}(t)$ for the vector $b_{j}(t)$. This formulation includes $\sigma_{1}(t)=\sigma_{2}(t)$, as studied in the literature (Trofino et al., 2009; Bolzern and Spinelli, 2004), as a special case. In addition to its theoretically interesting aspect, many application problems can be reduced to this form. As in switched linear systems, one important application for the above SAS is the design of multiple controllers for a single system. For example, given an LTI system $\dot{x}=A(t) x+B(t) u$, we consider the control input in the form of $u=K_{i}(t) x+\beta_{j}(t), i \in\left\{1, \ldots, \mathcal{N}_{1}\right\}$, $j \in\left\{1, \ldots, \mathcal{N}_{2}\right\}$. That is, we pre-design a bunch of state feedback gains $K_{i}(t)$, a bunch of time-varying vectors $\beta_{j}(t)$, and then choose a pair of $\left(K_{i}(t), \beta_{j}(t)\right)$ among them depending on different situations. In this case, setting $A_{i}(t)=A(t)+B(t) K_{i}(t)$ and $b_{j}(t)=B(t) \beta_{j}(t)$, we obtain the system (4). This remains true if we can renumber all pairs $(i, j)\left(i \in I_{\mathcal{N}_{1}}, j \in I_{\mathcal{N}_{2}}\right)$ to obtain an augmented SAS with $\mathcal{N}_{1} \times \mathcal{N}_{2}$ subsystems as used in the literature. However, since it is reasonable to endow the subsystem matrix and the affine vector with different potential and practical meanings, as described in the above mentioned switching controller design, we choose the system formulation (4).

In order to propose our switching strategy based on a convex combination of subsystems, we make the following assumption throughout this paper.

Assumption 1. There exist nonnegative functions $\lambda_{i}(t)$ $\left(i=1, \ldots, \mathcal{N}_{1}\right)$ satisfying $\sum_{i=1}^{\mathcal{N}_{1}} \lambda_{i}(t)=1$, such that the convex combined system

$$
\dot{x}(t)=A_{\lambda}(t) x(t), \quad A_{\lambda}(t)=\sum_{i=1}^{\mathcal{N}_{1}} \lambda_{i}(t) A_{i}(t)
$$

is uniformly asymptotically stable at the origin $x_{e}=0$.

Remark 2. Even when the system matrices $A_{i}(t)$ are constant, there is no globally effective method for checking whether or not Assumption 2 is true, i.e., finding nonnegative functions $\lambda_{i}(t)$ such that the real parts of the eigenvalues of $A_{\lambda}(t)$ are less than a negative scalar. However, we still have several sufficient or numerical methods for it. In the case of $\mathcal{N}_{1}=2$ or 3 , it is known that we can use a kind of griding method to find desirable $\lambda_{i}(t)$ such that $A_{\lambda}(t)$ is uniformly Hurwitz. In the case of $\mathcal{N}_{1}>3$, we may try to transform the system matrices into diagonal or triangular forms, which leads to less computation load. It is noted that due to the continuity of $A_{\lambda}$ in $\lambda$, if $A_{\lambda}$ is Hurwitz for some $\bar{\lambda}$, then there is a neighborhood region of $\bar{\lambda}$ where $A_{\lambda}(t)$ remains uniformly Hurwitz.

Now, we are ready to formulate our control problems as follows.

Quadratic stabilization (QS) (Whenever $w(t)=0$ ) Establish the condition and design the state feedback $\left(\sigma_{1}(x, t), \sigma_{2}(x, t)\right)$ and the output feedback $\left(\sigma_{1}(y, t)\right.$, $\left.\sigma_{2}(y, t)\right)$ such that the switched affine system (4) is quadratically stable at the origin.

Quadratic tracking (QT) (Whenever $w(t)=0$ ) Establish the condition and design the state feedback $\left(\sigma_{1}(x, t), \sigma_{2}(x, t)\right)$ and the output feedback $\left(\sigma_{1}(y, t)\right.$, $\left.\sigma_{2}(y, t)\right)$ such that the state of the switched affine system (4) tracks a desired trajectory $x_{e}(t)$ quadratically.

Quadratic $\mathcal{L}_{\mathbf{2}}$ gain $\gamma(\mathbf{Q L 2}-\gamma)$ (Whenever $w(t) \neq 0$ ) Establish the condition and design the state feedback $\left(\sigma_{1}(x, t), \sigma_{2}(x, t)\right)$ such that the switched affine system (4) is quadratically stable with $\mathcal{L}_{2}$ gain $\gamma$.

\section{Quadratic stabilization}

3.1. State feedback. In this section, we assume that the state $x(t)$ is available, and design a stabilizing switching law dependent on the system state such that the switched affine system is quadratically stable at the origin.

To achieve asymptotic stability of a switched nonlinear system at the origin, it has been shown that the necessary condition is that zero should be within the convex hull set of the subsystems' vector fields at $x=0$ (Xu and Zhai, 2005; Xu et al., 2008). This is described in the following assumption for the present switched affine system.

Assumption 2. There exist nonnegative functions $\mu_{j}(t)$ $\left(j=1, \ldots, \mathcal{N}_{2}\right)$ satisfying $\sum_{j=1}^{\mathcal{N}_{2}} \mu_{j}(t)=1$ such that

$$
b_{\mu}(t)=\sum_{j=1}^{\mathcal{N}_{2}} \mu_{j}(t) b_{j}(t)=0 .
$$

For notational simplicity, we will omit the time-varying symbol " $(t)$ " after the time-varying matrices and vectors $x(t), A_{i}(t), A_{\lambda}(t), b_{j}(t), b_{\mu}(t)$, $P(t), \lambda_{i}(t), \mu_{j}(t)$ and the like when no confusion arises. 
Theorem 1. Under Assumptions 1 and 2, the switched affine system (4) is quadratically stabilizable via state feedback.

Proof. Since the system $\dot{x}=A_{\lambda} x$ is uniformly asymptotically stable at $x_{e}=0$, there exists a real matrix function $P(t)$ and a scalar $\epsilon>0$ satisfying the differential LMI

$$
\dot{P}+A_{\lambda}^{\top} P+P A_{\lambda} \prec-\epsilon I_{n},
$$

where $P(t)$ is uniformly bounded and uniformly positive definite in the sense of satisfying $\alpha_{1} I_{n} \leq P(t) \leq \alpha_{2} I_{n}$, $0<\alpha_{1} \leq \alpha_{2}, \forall t \geq t_{0}$.

Use the matrix $P$ to define the switching law

$$
\begin{aligned}
& \left(\sigma_{1}(x, t), \sigma_{2}(x, t)\right) \\
& =\arg \min _{i \in I_{\mathcal{N}_{1}}, j \in I_{\mathcal{N}_{2}}}\left\{x^{\top}\left(A_{i}^{\top} P+P A_{i}\right) x+2 x^{\top} P b_{j}\right\} .
\end{aligned}
$$

In order to implement the above switching law clearly, we need to take care of the case when there are several indexes of subsystems obtained with (11). If these indexes include the present subsystem, that is,

$$
\begin{aligned}
\left(\sigma_{1}\left(x\left(t^{-}\right)\right), \sigma_{2}\left(x\left(t^{-}\right)\right)\right) & \\
\in \arg \min _{i \in I_{\mathcal{N}_{1}}, j \in I_{\mathcal{N}_{2}}}\left\{x ( t ) ^ { \top } \left(A_{i}^{\top} P\right.\right. & \left.+P A_{i}\right) x(t) \\
& \left.+2 x(t)^{\top} P b_{j}\right\},
\end{aligned}
$$

we choose to stay at the same subsystem without switching. Otherwise, we choose any subsystem index generated by 111). This rule is also applied to other switching laws later in this paper.

Under the switching law [11, the inequality

$$
\begin{aligned}
& x^{\top}\left(\dot{P}+A_{\sigma_{1}}^{\top} P+P A_{\sigma_{1}}\right) x+2 x^{\top} P b_{\sigma_{2}} \\
& \quad \leq x^{\top}\left(\dot{P}+A_{i}^{\top} P+P A_{i}\right) x+2 x^{\top} P b_{j}
\end{aligned}
$$

holds for all $i=1, \ldots, \mathcal{N}_{1}$ and $j=1, \ldots, \mathcal{N}_{2}$. Multiplying both sides of the above inequality by nonnegative functions $\lambda_{i} \mu_{j}$, and then adding all the inequalities results in

$$
\begin{aligned}
x^{\top} & \left(\dot{P}+A_{\sigma_{1}}^{\top} P+P A_{\sigma_{1}}\right) x+2 x^{\top} P b_{\sigma_{2}} \\
= & \sum_{i=1}^{\mathcal{N}_{1}} \sum_{j=1}^{\mathcal{N}_{2}} \lambda_{i} \mu_{j}\left(x^{\top}\left(\dot{P}+A_{\sigma_{1}}^{\top} P+P A_{\sigma_{1}}\right) x\right. \\
& \left.+2 x^{\top} P b_{\sigma_{2}}\right) \\
\leq & \sum_{i=1}^{\mathcal{N}_{1}} \sum_{j=1}^{\mathcal{N}_{2}} \lambda_{i} \mu_{j}\left(x^{\top}\left(\dot{P}+A_{i}^{\top} P+P A_{i}\right) x\right. \\
& \left.+2 x^{\top} P b_{j}\right)
\end{aligned}
$$

$$
\begin{aligned}
= & \sum_{i=1}^{\mathcal{N}_{1}} \lambda_{i}\left(x^{\top}\left(\dot{P}+A_{i}^{\top} P+P A_{i}\right) x\right. \\
& \left.+2 x^{\top} P b_{\mu}\right) \\
= & x^{\top}\left(\dot{P}+A_{\lambda}^{\top} P+P A_{\lambda}\right) x+2 x^{\top} P b_{\mu} \\
= & x^{\top}\left(\dot{P}+A_{\lambda}^{\top} P+P A_{\lambda}\right) x .
\end{aligned}
$$

Then, from (10) we obtain that

$$
x^{\top}\left(\dot{P}+A_{\sigma_{1}}^{\top} P+P A_{\sigma_{1}}\right) x+2 x^{\top} P b_{\sigma_{2}}<-\epsilon x^{\top} x .
$$

Since $\alpha_{1} I_{n} \leq P(t) \leq \alpha_{2} I_{n}$, we have

$$
x^{\top} P(t) x \leq \alpha_{2} x^{\top} x
$$

and thus

$$
\begin{aligned}
x^{\top}\left(\dot{P}+A_{\sigma_{1}}^{\top} P+P A_{\sigma_{1}}\right) x+2 x^{\top} & P b_{\sigma_{2}} \\
& <-\frac{\epsilon}{\alpha_{2}} x^{\top} P x
\end{aligned}
$$

for any nonzero $x$.

To apply Lyapunov's stability theory, we now consider the derivative of the Lyapunov function candidate $V(x, t)=x^{\top} P(t) x$ along the trajectories of the switched affine system (4) with $w=0$. Since the system state does not jump at the switching instants, we do not need to worry about the value change of $V(x, t)$ there. Thus, we only need to evaluate the derivative of $V(x, t)$ in each time interval for the activated subsystem as

$$
\begin{aligned}
\dot{V}(x, t) & =\frac{\mathrm{d}}{\mathrm{d} t} x^{\top} P x=\dot{x}^{\top} P x+x^{\top} P \dot{x}+x^{\top} \dot{P} x \\
& =x^{\top}\left(\dot{P}+A_{\sigma_{1}}^{\top} P+P A_{\sigma_{1}}\right) x+2 x^{\top} P b_{\sigma_{2}} \\
& <-\frac{\epsilon}{\alpha_{2}} V(x, t)
\end{aligned}
$$

for any nonzero $x$. This implies that the switched affine system (4) is quadratically stable at the origin.

Example 1. Consider the switched affine system (4) where the matrices and vectors are given by

$$
\begin{array}{ll}
A_{1}=\left[\begin{array}{cc}
-6 & 2 e^{0.1 t} \\
4 e^{0.5 t} & 2
\end{array}\right], & b_{1}=\left[\begin{array}{r}
-0.2 t^{2} \\
0.3 t^{2}
\end{array}\right], \\
A_{2}=\left[\begin{array}{cc}
1 & -e^{0.1 t} \\
-2 e^{0.5 t} & -3
\end{array}\right], & b_{2}=\left[\begin{array}{r}
0.6 t \\
-0.9 t
\end{array}\right] .
\end{array}
$$

It can be easily verified that both $A_{1}$ and $A_{2}$ are not uniformly stable (noticing $\left|A_{1}\right|=-12-8 e^{0.6 t}$ and $\left.\left|A_{2}\right|=-3-2 e^{0.6 t}\right)$, and that

$$
A_{1}+2 A_{2}=-4 I_{2}, \quad t \cdot b_{2}=-3 b_{1} .
$$

Thus, we have

$$
\lambda_{1}=\frac{1}{3}, \quad \lambda_{2}=\frac{2}{3},
$$




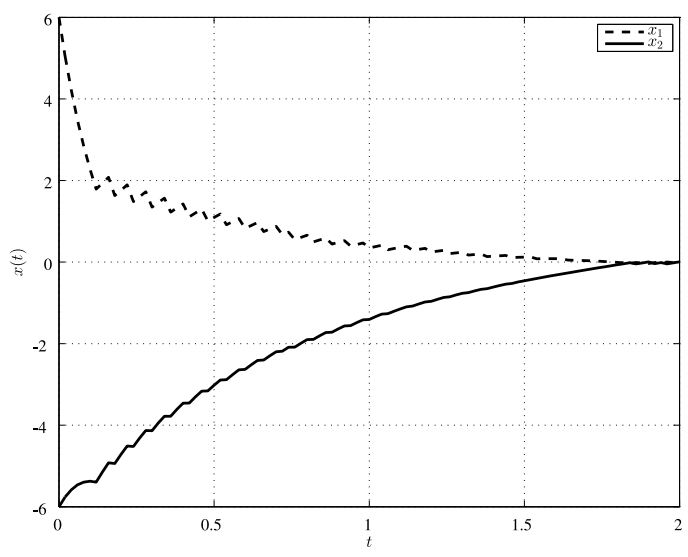

Fig. 1. Plot of the switched affine system under state feedback (Example 1).

$$
A_{\lambda}=-\frac{4}{3} I
$$

and

$$
\mu_{1}=\frac{3}{t+3}, \quad \mu_{2}=\frac{t}{t+3} .
$$

Since in this case $A_{\lambda}$ is a constant Hurwitz matrix, we can simply choose $P=I_{2}$.

Starting from the initial state $x_{0}=\left[\begin{array}{ll}6 & -6\end{array}\right]^{\top}$ and using the switching law (11), we obtain the state trajectory of the switched affine system depicted in Fig. 1.

3.2. Output feedback. Here, we assume that the system state $x(t)$ is not available for the switching law, and consider an observer to estimate the state. For this purpose, we adopt and modify the idea of the robust detectability condition (Packard, 1994; Feron, 1996) for the switched affine system, and propose a full dimensional Luenberger observer based on the solutions of a set of differential LMIs. Then, the switching law is constructed by using the observer's state and the LMIs' solution.

A natural extension of the Luenberger observer for the switched affine system (4) takes the form of

$$
\dot{\hat{x}}=A_{\sigma_{1}} \hat{x}+b_{\sigma_{2}}+L(t)(y-E \hat{x}),
$$

where $L(t)$ is the matrix function denoting the observer.

To design an observer for a single LTV (LTI) system, it is known that we need to pose a detectability or observability condition on the system matrix and the output matrix. Since we are here dealing with the switched affine system (4) and desire to obtain quadratic performance, we propose the following robust detectability condition, which is a revision of the existing one for linear time-invariant systems (Packard, 1994; Feron, 1996).
Assumption 3. There exist a uniformly bounded and uniformly positive definite matrix function $Q(t)$, a bounded matrix function $M(t)$ and a positive constant scalar $\xi$ satisfying

$$
\dot{Q}+A_{i}^{\top} Q+Q A_{i}-M E-E^{\top} M^{\top} \prec-\xi I_{n}
$$

for all $i=1, \ldots, \mathcal{N}_{1}$.

When (20) holds, we let $L=Q^{-1} M$ in the observer (19). Using the positive definite matrix $P$ and the estimated state $\hat{x}$, we define the output-dependent switching law by

$$
\begin{aligned}
& \left(\sigma_{1}(y, t), \sigma_{2}(y, t)\right) \\
= & \arg \min _{i \in I_{\mathcal{N}_{1}}, j \in I_{\mathcal{N}_{2}}}\left\{\hat{x}^{\top}\left(A_{i}^{\top} P+P A_{i}\right) \hat{x}+2 \hat{x}^{\top} P b_{j}\right\} .
\end{aligned}
$$

Theorem 2. Under Assumptions 1-3, the switched affine system (4) is quadratically stabilizable via output feedback, and the switching law is given by (21) and (19).

Proof. When $w=0$, the closed-loop system composed of the system (4) and the observer (19) is

$$
\left\{\begin{array}{l}
\dot{x}=A_{\sigma_{1}} x+b_{\sigma_{2}}, \\
\dot{\hat{x}}=A_{\sigma_{1}} \hat{x}+b_{\sigma_{2}}+L E(x-\hat{x}) .
\end{array}\right.
$$

Let $e(t)=\hat{x}(t)-x(t)$ to rewrite the above as

$$
\left\{\begin{array}{l}
\dot{\hat{x}}=A_{\sigma_{1}} \hat{x}+b_{\sigma_{2}}-L E e, \\
\dot{e}=\left(A_{\sigma_{1}}-L E\right) e .
\end{array}\right.
$$

Consider the Lyapunov function candidate

$$
V(\hat{x}, e)=\left[\begin{array}{c}
\hat{x} \\
e
\end{array}\right]^{\top}\left[\begin{array}{cc}
P & 0 \\
0 & \zeta Q
\end{array}\right]\left[\begin{array}{l}
\hat{x} \\
e
\end{array}\right],
$$

where $\zeta$ is a positive scalar to be adjusted. Then, the derivative of $V(\hat{x}, e)$ along the solutions of the closed-loop system (23) is computed and evaluated as

$$
\begin{aligned}
\dot{V}(\hat{x}, e)= & \frac{\mathrm{d}}{\mathrm{d} t}\left(\hat{x}^{\top} P \hat{x}+\zeta e^{\top} Q e\right) \\
= & \hat{x}^{\top}\left(\dot{P}+A_{\sigma_{1}}^{\top} P+P A_{\sigma_{1}}\right) \hat{x} \\
& +2 \hat{x}^{\top} P b_{\sigma_{2}}-2 \hat{x}^{\top} P L E e \\
& +\zeta e^{\top}\left(\dot{Q}+\left(A_{\sigma_{1}}-L E\right)^{\top} Q\right. \\
& \left.+Q\left(A_{\sigma_{1}}-L E\right)\right) e .
\end{aligned}
$$

Comparing the switching strategy in (21) and the one in (11), we obtain, similarly to the proof of Theorem 1, that

$$
\begin{aligned}
\hat{x}^{\top}\left(\dot{P}+A_{\sigma_{1}}^{\top} P+\right. & \left.P A_{\sigma_{1}}\right) \hat{x}+2 \hat{x}^{\top} P b_{\sigma_{2}} \\
& \leq \hat{x}^{\top}\left(\dot{P}+A_{\lambda}^{\top} P+P A_{\lambda}\right) \hat{x} .
\end{aligned}
$$


Moreover, using the relation $L=Q^{-1} M$ in 20), we obtain

$$
\begin{aligned}
\dot{Q}+ & \left(A_{\sigma_{1}}-L E\right)^{\top} Q+Q\left(A_{\sigma_{1}}-L E\right) \\
& =\dot{Q}+A_{\sigma_{1}}^{\top} Q+Q A_{\sigma_{1}}-M E-E^{\top} M^{\top} \\
& \prec-\xi I_{n} .
\end{aligned}
$$

Substituting (26) and 27) into (25) gives

$$
\begin{aligned}
\dot{V}(\hat{x}, e) \leq & \hat{x}^{\top}\left(\dot{P}+A_{\lambda}^{\top} P+P A_{\lambda}\right) \hat{x} \\
& -2 \hat{x}^{\top} P L E e-\zeta \xi e^{\top} e \\
\leq & -\frac{\epsilon}{\alpha_{2}} \hat{x}^{\top} P \hat{x}-2 \hat{x}^{\top} P L E e-\zeta \xi e^{\top} e \\
= & -\left[\begin{array}{c}
\hat{x} \\
e
\end{array}\right]^{\top} \mathcal{P}(\zeta, t)\left[\begin{array}{l}
\hat{x} \\
e
\end{array}\right],
\end{aligned}
$$

where

$$
\mathcal{P}(\zeta, t)=\left[\begin{array}{cc}
\frac{\epsilon}{\alpha_{2}} P & P L E \\
(P L E)^{\top} & \zeta \xi I_{n}
\end{array}\right] .
$$

Since $P$ and $Q$ are uniformly bounded and positive definite, $M$ is bounded and $L$ is also uniformly bounded. Then we can always choose sufficiently large $\zeta>0$ such that $\mathcal{P}(\zeta, t)$ is uniformly positive definite, and thus $\dot{V}(\hat{x}, e)$ is uniformly negative. Actually, using Schur's Lemma, we can achieve this by choosing $\zeta$ such that

$$
\begin{aligned}
\zeta I_{n} & \succ \frac{\alpha_{2}}{\epsilon \xi} E^{\top} L^{\top} P L E \\
& \Longleftrightarrow \zeta>\frac{\alpha_{2}}{\epsilon \xi} \sup _{t \geq 0} \lambda_{\max }\left(E^{\top} L^{\top} P L E\right) .
\end{aligned}
$$

This implies that the closed-loop system is quadratically stable.

Remark 3. As can be seen from the above proof, the differential matrix inequality (20) is a robust detectability condition for the linear time-variant system, which ensures convergence of the error $e(t)$ between the estimate $\hat{x}(t)$ and the true state $x(t)$ to zero.

Example 2. We revisit the system in Example 1, but now assume that the state information is not available, and the measurement output matrix is $E=\left[\begin{array}{ll}1 & 1\end{array}\right]$.

First, we need to check Assumption 3 by solving the differential LMIs with respect to $Q(t)$ and $M(t)$. However, it is well known that there is no efficient and practical approach to solving a differential matrix inequality globally. Here, we propose approximately solving the inequality online by choosing the second-order Taylor expansion of the matrix variable such as $Q(t)=Q_{0}+t Q_{1}+t^{2} Q_{2}$, where $Q_{0}, Q_{1}$ and $Q_{2}$ are symmetric. Although such kind of approximation does not guarantee uniform boundedness of the matrix
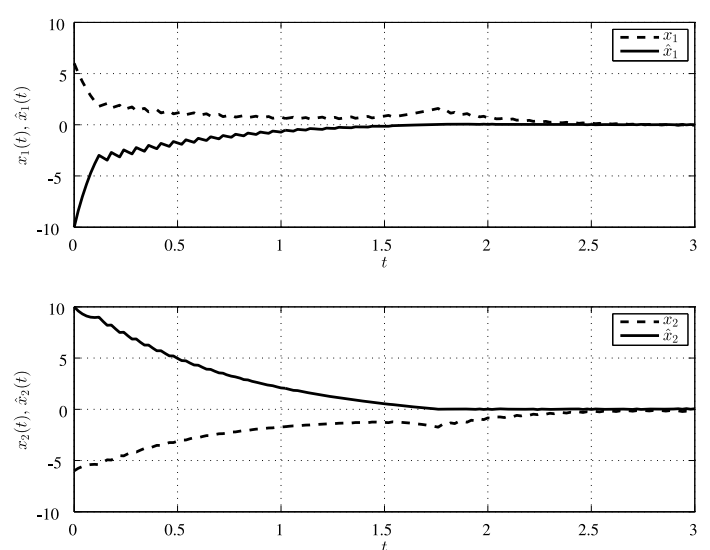

Fig. 2. Plot of the switched affine system under output feedback (Example 2).

variables, we can check the state trajectories, and increase the order of Taylor expansions when necessary.

The observer gain matrix is computed by $L(t)=$ $Q^{-1}(t) M(t)$. Using this gain matrix in the observer (19) and activating the switching law (21) for the switched affine system with the initial states $x(0)=\left[\begin{array}{ll}6 & -6\end{array}\right]^{\top}$, $\hat{x}(0)=\left[\begin{array}{ll}-10 & 10\end{array}\right]^{\top}$, we obtain the state trajectories of the whole system depicted in Fig. 2.

\section{Quadratic tracking}

In this section, we release Assumption 2 and consider the tracking control problem for the switched affine system (4). More precisely, we shall ask what state trajectory the switched affine system can track quadratically, and how to design the switching law for given trackable trajectory.

Let us assume that the reference input (trajectory) is $r(t)$, and define $z(t)=x(t)-r(t)$ as the tracking error. Then substituting $x(t)=z(t)+r(t)$ into the original switched system leads to

$$
\dot{z}=A_{\sigma_{1}} z+\left(A_{\sigma_{1}} r+b_{\sigma_{2}}-\dot{r}\right) .
$$

Since $z(t)$ is the difference between the state and the reference input, we also need to consider how to design the switching laws based on the system state and the measurement output.

4.1. State feedback. When the state $x$ is available, $z=$ $x-r$ is also available. In this case, we define the switching law

$$
\begin{aligned}
\left(\sigma_{1}(z, t), \sigma_{2}(z, t)\right) & \\
=\arg \min _{i \in I_{\mathcal{N}_{1}}, j \in I_{\mathcal{N}_{2}}} & \left\{z^{\top}\left(A_{i}^{\top} P+P A_{i}\right) z\right. \\
+ & \left.2 z^{\top} P\left(A_{i} r+b_{j}\right)\right\},
\end{aligned}
$$


where $P$ is the same matrix function as before, i.e., the one satisfying the differential LMI (10). Then, under the switching law (32), we obtain

$$
\begin{aligned}
& z^{\top}\left(\dot{P}+A_{\sigma_{1}}^{\top} P+P A_{\sigma_{1}}\right) z+2 z^{\top} P\left(A_{\sigma_{1}} r+b_{\sigma_{2}}-\dot{r}\right) \\
& \quad \leq z^{\top}\left(\dot{P}+A_{i}^{\top} P+P A_{i}\right) z+2 z^{\top} P\left(A_{i} r+b_{j}-\dot{r}\right)
\end{aligned}
$$

for all $i=1, \ldots, \mathcal{N}_{1}$ and $j=1, \ldots, \mathcal{N}_{2}$. Multiplying both sides of the above inequality by nonnegative functions $\lambda_{i} \mu_{j}$, and then adding all the inequalities results in

$$
\begin{aligned}
z^{\top} & \left(\dot{P}+A_{\sigma_{1}}^{\top} P+P A_{\sigma_{1}}\right) z+2 z^{\top} P\left(A_{\sigma_{1}} r+b_{\sigma_{2}}-\dot{r}\right) \\
& \leq z^{\top}\left(\dot{P}+A_{\lambda}^{\top} P+P A_{\lambda}\right) z+2 z^{\top} P\left(A_{\lambda} r+b_{\mu}-\dot{r}\right) \\
& \leq-\frac{\epsilon}{\alpha_{2}} V(z, t)+2 z^{\top} P\left(A_{\lambda} r+b_{\mu}-\dot{r}\right) .
\end{aligned}
$$

Similarly to the hitherto lead discussion, we consider the Lyapunov function candidate $V(z, t)=z^{\top} P(t) z$ and evaluate the derivative of $V(z, t)$ along the trajectories of the error system 31 with $w=0$ by

$$
\dot{V}(z, t)<-\frac{\epsilon}{\alpha_{2}} V(z, t)+2 z^{\top} P\left(A_{\lambda} r+b_{\mu}-\dot{r}\right)
$$

for any nonzero $z$. Therefore, if the reference trajectory is defined by

$$
\dot{r}=A_{\lambda} r+b_{\mu}, \quad r\left(t_{0}\right)=r_{0}: \text { arbitrary, }
$$

then

$$
\dot{V}(z, t)<-\frac{\epsilon}{\alpha_{2}} V(z, t)
$$

and the tracking error $z(t)$ converges to zero quadratically.

We summarize the above discussion in the following theorem.

Theorem 3. Under Assumption 1, the state of the switched affine system (4) quadratically tracks any reference trajectory $r(t)$ generated by (36) with the switching law (32).

Remark 4. If Assumption 2 holds, i.e., $b_{\mu}=0$, then the differential equation (36) shrinks into $\dot{r}=A_{\lambda} r$. If we furthermore choose the initial value of $r$ being zero, then $r$ is zero for all $t$. This is the case of quadratic stabilization we studied in the previous section. In this sense, the discussion in this section is an extension to that in the previous section.

Remark 5. If the transition matrix for $\dot{r}=A_{\lambda} r$ is $\Phi\left(t, t_{0}\right)$, then the trackable trajectories are given by

$$
r(t)=\Phi\left(t, t_{0}\right) r_{0}+\int_{t_{0}}^{t} \Phi(t, \tau) b_{\mu}(\tau) \mathrm{d} \tau
$$

where the initial value $r_{0}$ and the functions $\mu_{j}$ 's can be used to design a desired reference trajectory. More precisely, since

$$
b_{\mu}=\sum_{j=1}^{\mathcal{N}_{2}} \mu_{j} b_{j}, \quad \mu_{j} \geq 0, \quad \sum_{j=1}^{\mathcal{N}_{2}} \mu_{j}=1,
$$

we obtain

$$
\begin{aligned}
r & =\sum_{j=1}^{\mathcal{N}_{2}} \mu_{j} r_{j}, \\
r_{j} & =\Phi\left(t, t_{0}\right) r_{0}+\int_{t_{0}}^{t} \Phi(t, \tau) b_{j}(\tau) \mathrm{d} \tau .
\end{aligned}
$$

In other words, $r$ is always generated by the polytope whose extreme points are known time-varying vectors $r_{j}$, $j=1, \ldots, \mathcal{N}_{2}$. This observation is useful when one wishes to adjust the tracking trajectory online.
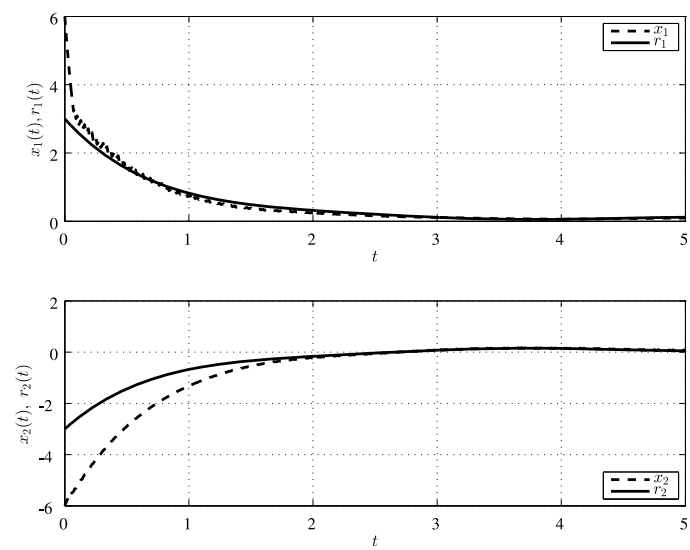

Fig. 3. Plot of the switched affine system with the reference trajectory (Example 3).

Example 3. Change the affine vectors of the switched affine system in Example 1 to

$$
b_{1}=\left[\begin{array}{r}
0.2 \\
0
\end{array}\right], \quad b_{2}=\left[\begin{array}{r}
0 \\
0.3
\end{array}\right] .
$$

Since $A_{1}$ and $A_{2}$ are the same as in Example 1, $A_{\lambda}$ and $P$ do not change. Setting $\mu_{1}=\sin ^{2} t, \mu_{2}=\cos ^{2} t$ and assuming the initial values of $r_{1}, r_{2}$ are 3 and -3 , respectively, from (36) we obtain that

$$
\dot{r}=-\frac{4}{3} r+\left[\begin{array}{l}
0.2 \sin ^{2} t \\
0.3 \cos ^{2} t
\end{array}\right], \quad r(0)=\left[\begin{array}{r}
3 \\
-3
\end{array}\right] .
$$

Using the same $P=I_{2}$, the same initial value of $x$ and the switching law (32) for the switched affine system, we plot the state and reference trajectories in Fig. 3, which shows the tracking performance is obtained. 
4.2. Output feedback. As in the previous section, when the system state $x(t)$ is not available for the switching strategy, we construct the robust observer (19) under Assumption 3. Then we define $\hat{z}(t)=\hat{x}(t)-r(t)$, and modify the switching law (32) to

$$
\begin{aligned}
\left(\sigma_{1}(y, t), \sigma_{2}(y, t)\right) & \\
=\arg \min _{i \in I_{\mathcal{N}_{1}}, j \in I_{\mathcal{N}_{2}}} & \left\{\hat{z}^{\top}\left(A_{i}^{T} P+P A_{i}\right) \hat{z}\right. \\
+ & \left.2 \hat{z}^{\top} P\left(A_{i} r+b_{j}\right)\right\} .
\end{aligned}
$$

Combining the proofs for Theorems 2 and 3, we can easily prove the next result, but omit that for brevity.

Theorem 4. Under Assumptions 1 and 3, the state of the switched affine system (4) quadratically tracks any reference trajectory $r(t)$ generated by (36) with the switching law (40) and (19).

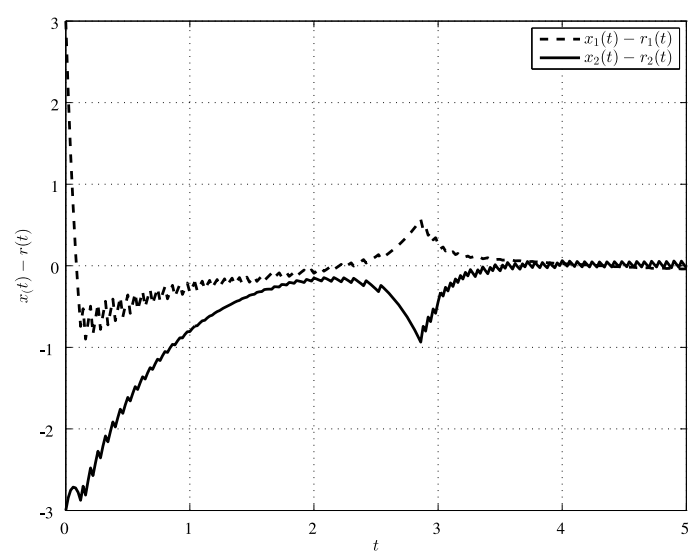

Fig. 4. Tracking error of the switched affine system with the reference trajectory (Example 4).

Example 4. We consider the same switched affine system as in Example 3, but now assume that the state information is not available, and the measurement output matrix is the same as in Example 2.

Using the same method as in Example 2, we compute the gain matrix function of the observer (19) and activate the switching law (40) for the switched affine system with the initial states $x(0)=\left[\begin{array}{ll}6 & -6\end{array}\right]^{\top}, \hat{x}(0)=$ $\left[\begin{array}{ll}-10 & 10\end{array}\right]^{\top}$, and $r(0)=\left[\begin{array}{ll}3 & -3\end{array}\right]^{\top}$. The tracking error between the system state and the reference trajectory is depicted in Fig. 4, which shows that the tracking performance is obtained.

\section{Quadratic $\mathcal{L}_{2}$ gain analysis}

In this section, we consider $\mathcal{L}_{2}$ gain analysis problem for the switched affine system (4). For $\mathcal{H}_{\infty}$ disturbance attenuation, $\mathcal{L}_{2}$ gain analysis problem has been proposed and studied for switched linear systems (Zhai et al., 2001). As was already mentioned in Introduction, we used the idea of the convex combination of subsystems to establish the switching law which achieved quadratic stabilization with a desired $\mathcal{L}_{2}$ gain (Zhai, 2012). The above existing results are for switched linear systems, and here we aim to extend the results of Zhai (2012) to the switched affine system (4).

To deal with quadratic stabilization with the $\mathcal{L}_{2}$ gain property, we need to strengthen Assumption 1 as in Assumption 4, which requires stability and the desired $\mathcal{L}_{2}$ gain property of the combined system described by the quadruple $\left(A_{\lambda}, B, C, D\right)$.

Assumption 4. There exist nonnegative functions $\lambda_{i}$ $\left(i=1, \ldots, \mathcal{N}_{1}\right)$ satisfying $\sum_{i=1}^{\mathcal{N}_{1}} \lambda_{i}=1$ such that the convex combined system

$$
\left\{\begin{aligned}
\dot{x} & =A_{\lambda} x+B w \\
z & =C x+D w
\end{aligned}\right.
$$

is uniformly asymptotically stable at the origin, and the $\mathcal{L}_{2}$ gain from $w$ to $z$ is less than a positive scalar $\gamma$.

Theorem 5. Under Assumptions 2 and 4, the switched affine system (4) is quadratically stabilizable with $\mathcal{L}_{2}$ gain $\gamma$ via state feedback.

Proof. According to Lemma 1, under Assumption 4, there exists a uniformly bounded and positive definite matrix function $P$ such that

$$
\left[\begin{array}{cc}
\dot{P}+A_{\lambda}^{\top} P+P A_{\lambda}+C^{\top} C & P B+C^{\top} D \\
B^{\top} P+D^{\top} C & -\gamma^{2} I+D^{\top} D
\end{array}\right]
$$

is uniformly negative definite, and thus there always exists a positive scalar $\epsilon$ such that

$$
\left[\begin{array}{cc}
\dot{P}+A_{\lambda}^{\top} P+P A_{\lambda}+C^{\top} C+\epsilon P & P B+C^{\top} D \\
B^{\top} P+D^{\top} C & -\gamma^{2} I+D^{\top} D
\end{array}\right]
$$

is still uniformly negative definite. Using the positive definite matrix $P$, we adopt the switching law defined in (11). Then, as shown in the proof of Theorem 1, (12) and (13) hold.

The derivative of the Lyapunov function candidate $V(x, t)=x^{\top} P x$ along the trajectories of the switched affine system (4) is computed and evaluated as

$$
\begin{aligned}
\dot{V}(x, t)= & \dot{x}^{\top} P x+x^{\top} P \dot{x}+x^{\top} \dot{P} x \\
= & x^{\top}\left(\dot{P}+A_{\sigma_{1}}^{\top} P+P A_{\sigma_{1}}\right) x+2 x^{\top} P b_{\sigma_{2}} \\
& \quad+w^{\top} B^{\top} P x+x^{\top} P B w .
\end{aligned}
$$


Using (13) and then (43) in the above, we obtain

$$
\begin{aligned}
\dot{V} & (x, t) \\
\leq & x^{\top}\left(\dot{P}+A_{\lambda}^{\top} P+P A_{\lambda}\right) x+w^{\top} B^{\top} P x+x^{\top} P B w \\
= & {\left[\begin{array}{c}
x \\
w
\end{array}\right]^{\top}\left[\begin{array}{cc}
\dot{P}+A_{\lambda}^{\top} P+P A_{\lambda} & P B \\
B^{\top} P & 0
\end{array}\right] } \\
& \times\left[\begin{array}{c}
x \\
w
\end{array}\right] \\
< & -\left[\begin{array}{c}
x \\
w
\end{array}\right]^{\top}\left[\begin{array}{cc}
C^{\top} C+\epsilon P & C^{\top} D \\
D^{\top} C & -\gamma^{2} I+D^{\top} D
\end{array}\right] \\
& \times\left[\begin{array}{c}
x \\
w
\end{array}\right] \\
= & -\epsilon V(x, t)-z^{\top} z+\gamma^{2} w^{\top} w
\end{aligned}
$$

for any nonzero $x$ and any $w(t) \in \mathcal{L}_{2}[0, \infty)$. This completes the proof.

Remark 6. It is to be noted that the switching laws (11), (21), (32) and (40) are called the minimum (energy) rule (van der Schaft and Schumacher, 2000), and theoretically they may result in the so-called "chattering" or "Zeno" phenomena (switchings occur an infinite number of times on a finite time interval), which are not desired in real applications. To get rid of the possibility of this trouble, we propose to revise the hybrid switching rule (see, e.g., Bolzern and Spinelli, 2004; Luis-Delgado et al., 2017) for our switched system. More precisely, assuming the present activated subsystem is $\mathcal{S}_{i_{0} j_{0}}\left(\sigma_{1}=i_{0}, \sigma_{2}=j_{0}\right)$, we do not switch to other subsystems until the tolerance bound (essentially the same as in (11))

$$
\begin{gathered}
x^{\top}\left(A_{i 0}^{\top} P+P A_{i 0}\right) x+2 x^{\top} P b_{j 0} \\
<x^{\top}\left(A_{i}^{\top} P+P A_{i}\right) x+2 x^{\top} P b_{j}, \\
\forall i \neq i_{0}, \quad \forall j \neq j_{0},
\end{gathered}
$$

is violated. This is based on the observation that the above inequality holds on a nonzero time interval. Furthermore, for the purpose of real applications, we may set a small enough lower bound $\rho_{\text {off }}$ for $\|x\|$ such that switching is stopped when $\|x\| \leq \rho_{\text {off }}$, if necessary.

\section{Concluding remarks}

In this paper, we have extended the concept of the convex combination of subsystems for switched linear systems to quadratic stabilization, tracking and $\mathcal{L}_{2}$ gain analysis for switched affine time-varying systems. Different from the literature, we have formulated a new type of switched affine systems, where both subsystem matrices and affine vectors are switched independently and depend on time $t$. Assuming that no single subsystem has the desired quadratic stability, we have shown that if a convex combination of subsystem matrices is uniformly Hurwitz and another convex combination of affine vectors is zero, then we can design a state-dependent switching law (state feedback) and an output-dependent switching law (output feedback) such that the entire switched system is quadratically stable. The result has been extended to tracking and $\mathcal{L}_{2}$ gain analysis under state feedback in a natural manner.

Several issues are in progress. First, the question of output-dependent switching laws (output feedback) for $\mathcal{L}_{2}$ gain analysis of switched affine systems, with less conservative conditions, remains open. Secondly, switched discrete-time affine systems (Zhai et al., 2007) and asynchronous switching (Xiang et al., 2010) among affine systems could be practical and significant extensions of the present study. Furthermore, the discussion in this paper may be applied to design a cooperative control algorithm for multi-agent systems described by affine systems with switching topologies (Zhai, 2015; Zhai and Huang, 2015).

\section{Acknowledgment}

We would like to express our thanks to the editor and the anonymous reviewers for their detailed and precious comments, which were very helpful in revising the manuscript.

This research has been supported in part by the Japan Ministry of Education, Sciences and Culture under Grants-in-Aid for Scientific Research (C) 21560471. It has been jointly aided by the Research Project Supported by the Shanxi Scholarship Council of China under the grant 2015-044, the Fundamental Research Project of Shanxi Province under the grant 2015021085, and the National Science Foundation of China under the grant 61603268.

\section{References}

Allerhand, L.I. and Shaked, U. (2011). Robust stability and stabilization of linear switched systems with dwell time, IEEE Transactions on Automatic Control 56(2): 381-386.

Bolzern, P. and Spinelli, W. (2004). Quadratic stabilization of a switched affine system about a nonequilibrium point, Proceedings of the American Control Conference, Boston, MA, USA, pp. 3890-3895.

Branicky, M.S. (1998). Multiple Lyapunov functions and other analysis tools for switched and hybrid systems, IEEE Transactions on Automatic Control 43(4): 475-482.

Deaecto, G.S. (2016). Dynamic output feedback $H_{\infty}$ control of continuous-time switched affine systems, Automatica 71(1): 44-49. 
Deaecto, G.S. and Santos, G.C. (2015). State feedback $H_{\infty}$ control design of continuous-time switched affine systems, IET Control Theory \& Applications 9(10): 1511-1516.

DeCarlo, R., Branicky, M.S., Pettersson, S. and Lennartson, B. (2000). Perspectives and results on the stability and stabilizability of hybrid systems, Proceedings of the IEEE 88(7): 1069-1082.

Feron, E. (1996). Quadratic stability of switched systems via state and output feedback, MIT Technical Reports CICSP468, MIT, Cambridge, MA.

Hetel, L. and Fridman, E. (2013). Robust sampled-data control of switched affine systems, IEEE Transactions on Automatic Control 58(11): 2922-2928.

Leth, J. and Wisniewski, R. (2014). Local analysis of hybrid systems on polyhedral sets with state-dependent switching, International Journal of Applied Mathematics and Computer Science 24(2): 341-355, DOI: 10.2478/amcs-2014-0026.

Liberzon, D. (2003). Switching in Systems and Control, Birkhäuser, Boston, MA.

Liberzon, D. and Morse, A.S. (1999). Basic problems in stability and design of switched systems, IEEE Control Systems Magazine 19(5): 59-70.

Luis-Delgado, J.D., Al-Hadithi, B.M. and Jiménez, A. (2017). A novel method for the design of switching surfaces for discretized MIMO nonlinear systems, International Journal of Applied Mathematics and Computer Science 27(1): 5-17, DOI: 10.1515/amcs-2017-0001.

Packard, A. (1994). Gain scheduling via linear fractional transformation, Systems \& Control Letters 22(2): 79-92.

Pettersson, S. and Lennartson, B. (2002). Hybrid system stability and robustness verification using linear matrix-inequalities, International Journal of Control 75(16-17): 1335-1355.

Scharlau, C.C., de Oliveira, M.C., Trofino, A. and Dezuo, T.J.M. (2014). Switching rule design for affine switched systems using a max-type composition rule, Systems \& Control Letters 68(1): $1-8$.

Skelton, R.E., Iwasaki, T. and Grigoriadis, K.M. (1998). A Unified Algebraic Approach to Linear Control Design, Taylor \& Francis, London.

Sun, Z. and Ge, S.S. (2005). Switched Linear Systems: Control and Design, Springer, London.

Trofino, A., Assmann, D., Scharlau, C.C. and Coutinho, D.F. (2009). Switching rule design for switched dynamic systems with affine vector fields, IEEE Transactions on Automatic Control 54(9): 2215-2222.

van der Schaft, A. and Schumacher, H. (2000). An Introduction to Hybrid Dynamical Systems, Springer, London.

Wicks, M.A., Peleties, P. and DeCarlo, R. A. (1998). Switched controller design for the quadratic stabilization of a pair of unstable linear systems, European Journal of Control 4(2): 140-147.
Xiang, W. (2016). Necessary and sufficient condition for stability of switched uncertain linear systems under dwell-time constraint, IEEE Transactions on Automatic Control 61(11): 3619-3624.

Xiang, W. and Xiao, J. (2014). Stabilization of switched continuous-time systems with all modes unstable via dwell time switching, Automatica 50(3): 940-945.

Xiang, Z., Wang, R. and Chen, Q. (2010). Fault tolerant control of switched nonlinear systems with time delay under asynchronous switching, International Journal of Applied Mathematics and Computer Science 20(3): 497-506, DOI: 10.2478/v10006-010-0036-0.

Xu, X. and Zhai, G. (2005). Practical stability and stabilization of hybrid and switched systems, IEEE Transactions on Automatic Control 50(11): 1897-1903.

Xu, X., Zhai, G. and He, S. (2008). On practical asymptotic stabilizability of switched affine systems, Nonlinear Analysis: Hybrid Systems 2(1): 196-208.

Yoshimura, V.L., Assuncao, E., da Silva, E.R.P., Teixeira, M.C.M. and Mainardi Jr., E.I. (2013). Observer-based control design for switched affine systems and applications to DC-DC converters, Journal of Control, Automation and Electrical Systems 24(4): 535-543.

Zhai, G. (2001). Quadratic stabilizability of discrete-time switched systems via state and output feedback, Proceedings of the Conference on Decision and Control, Orlando, Florida, FL, USA, pp. 2165-2166.

Zhai, G. (2012). Quadratic stabilizability and $H_{\infty}$ disturbance attenuation of switched linear systems via state and output feedback, Proceedings of the Conference on Decision and Control, Maui, HI, USA, pp. 1935-1940.

Zhai, G. (2015). A generalization of the graph Laplacian with application to a distributed consensus algorithm, International Journal of Applied Mathematics and Computer Science 25(2): 353-360, DOI: 10.1515/amcs-2015-0027.

Zhai, G., Hu, B., Yasuda, K. and Michel, A.N. (2001). Disturbance attenuation properties of time-controlled switched systems, Journal of The Franklin Institute 338(7): 765-779.

Zhai, G. and Huang, C. (2015). A note on basic consensus problems in multi-agent systems with switching interconnection graphs, International Journal of Control 88(3): 631-639.

Zhai, G., Lin, H. and Antsaklis, P.J. (2003). Quadratic stabilizability of switched linear systems with polytopic uncertainties, International Journal of Control 76(7): 747-753.

Zhai, G., Xu, X., Lin, H. and Liu, D. (2007). Extended Lie algebraic stability analysis for switched systems with continuous-time and discrete-time subsystems, International Journal of Applied Mathematics and Computer Science 17(4): 447-454, DOI: 10.2478/v100006-007-0036-x. 


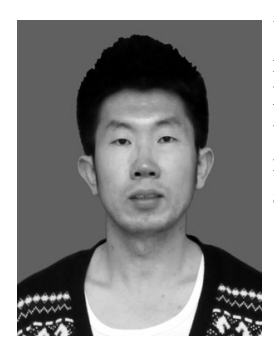

Wenzhi Li received his BSc degree in mathematics from Datong University, China, in 2015. $\mathrm{He}$ is now an MSc candidate at the Taiyuan University of Technology, China. His main research interests are in the stability analysis of switched affine systems.

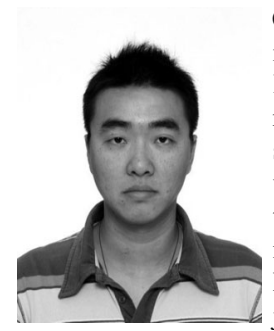

Chi Huang received his MSc and $\mathrm{PhD}$ degrees in mathematics from the City University of Hong Kong, Kowloon, Hong Kong, in 2009 and 2013, respectively. He is currently an associate professor at the College of Mathematics, Taiyuan University of Technology, China. His research interests include multi-agent systems, complex dynamical networks and Boolean networks. Dr. Huang has published more than 30 academic journal papers. He was the recipient of the Best Paper Award at the 8th Asian Control Conference (ASCC2011).

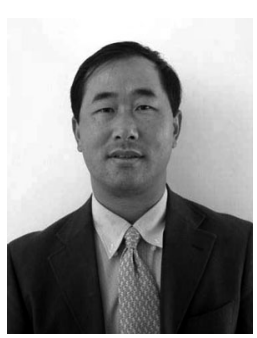

Guisheng Zhai received the BSc degree from Fudan University, China, in 1988, and the ME and $\mathrm{PhD}$ degrees, both in systems science, from Kobe University, Japan, in 1993 and 1996, respectively. After two years' industrial experience, Dr. Zhai moved to Wakayama University, Japan, in 1998, and then to Osaka Prefecture University, Japan, in 2004. In 2010, he joined the Shibaura Institute of Technology, Japan, where he is currently a professor of mathematical sciences. His research interests include large scale and decentralized control systems, robust control, switched systems and switching control, networked control systems, neural networks and signal processing, multiagent intelligent systems, etc. Dr. Zhai has published more than 100 academic journal papers and 160 peer-reviewed international conference papers.

Received: 8 August 2017

Revised: 21 November 2017

Re-revised: 6 February 2018 Accepted: 21 April 2018 